\title{
Analytische Studie über die paracelsische Ursprache
}

\author{
Von J.Strebel, Luzern
}

Karl Bittel, Überlingen, schreibt in seiner Kurzbiographie «in Selbstzeugnissen» (Reclam, 1943) zur grundsätzlichen Sprachfassung und Schreibweise Hohenheims: Die Sprache von Paracelsus ist durchaus eigenwillig; viele seiner Worte sind Neuschöpfungen der deutschen (und, fügen wir hinzu, der lateinischen und griechischen) Sprache. Von originaler Schrift aus seiner Hand ist außer ein paar Briefen und Konsilien überhaupt nichts erhalten. Die Diktate an seine Schreiber sowie Schülernachschriften sinḍ ohne Originalwert; das meist in späteren Abschriften Überlieferte ist es noch weniger. Auch das Wenige, was unter seinen Augen zum Druck kam, ist willkürlich gefaßt, wie Vergleiche beweisen. Wir besitzen also nur eine sehr unvollkommene Vorstellung der paracelsischen Sprache, die ein derbes Alemannisch-Schwäbisch gewesen ist.

Johannes Huserus Brisgoius (von Waldkirch bei Freiburg im Br. gebürtig, Schüler des Handschriftensammlers Joannes Scultetus Montanus, Arzt in Hirschberg, praktizierte zu Glogau in Schlesien, aktivster Paracelsist, der 1589/1591 die wichtigste und beste erste Gesamtausgabe in Quart bei Waldkirch Basel edierte) wählte bereits eine für das 16. Jahrhundert gemäße normalisierte Schreibung und der Zweitherausgeber Prof. Karl Sudhoff, der ursprünglich Germanist werden wollte, hat für seine Zweitausgabe der "Sämtlichen Werke» eine eigene, besser lesbare Orthographie rekonstruiert, wie er im I. Band, S. XXX, auseinandersetzt.

Der Literarhistoriker Friedrich Gundolf hat sich 1928 nicht so sehr philologisch als sprach- und sinnkritisch mit Paracelsus befaßt, wobei ihm neben viel Schwulitäten und Übersteigerungen wie Durchdeutschung, Entformelung, Entdinglichung, Entichung, Dingverformelung usw. einige gute Charakteristiken gelangen wie die folgenden: Luther hat keine größere Fülle anschaulicher Gleichnisse. Große Naturforscher, die als solche die deutsche Sprache gehoben, gibt es weniger als große Geschichtschreiber: bis in Goethes Jahrhundert ist Paracelsus der einzige. An scharfer, runder, tiefer Beobachtung, an wissenschaftlicher Sinnlichkeit kann es Hohenheim mit jedem Modernen aufnehmen. An Fülle wie an Stärke wird er von keinem Deutschen des 16. Jahrhunderts, von wenigen aller Zeiten, übertroffen. Unter den deutschen Naturforschern ist ihm, von Goethe immer abgesehen, nur Kepler an persönlicher Leuchtkraft gleich, auch nicht Gauß und Humboldt, Haller und Johannes Müller, Liebig und Helmholtz 
oder gar Virchow. Diesen literarhistorischen Essay Gundolfs kritisiert einer der tiefsten Paracelsus-Kenner, Will-Erich Peuckert, in seiner Paracelsus-Biographie 1943, S. 405, folgendermaßen: Arbeiten wie etwa die Gundolfs, der die Hartmannsche Biographie in seine Sprache umschrieb, ohne je in die Arbeit an Paracelsus selbst eingetreten zu sein, können nichts nützen, und ehe nicht eine lange geduldige Forschung vorangegangen ist, gereichen sie nur zu Schaden. Jede Arbeit an Paracelsus muß von seinem Werk ausgehen, wie es hier in einer kleinen Studie über die Ursprache Hohenheims anhand eines einzigartigen, in der Schweiz von Paracelsi eigener Hand geschriebenen und nie von Kopistenhand verwässerten Dokumentes geschehen soll.

Wenn Karl Bittel l. c. S. 140 schreibt, daß zu Lebzeiten Hohenheims nicht mehr als die beiden Syphilisschriften und der Doppelband der «Großen Wundarznei» erschienen seien, so muß er dahin ergänzt werden, daß doch die Badeverwaltung von Pfäfers ein großes Interesse daran hatte, Paracelsi Bäderschrift bald nach deren Niederschrift 1535 in (Zürich oder) St. Gallen drucken zu lassen. Solche Originaldrucke sind jetzt noch im Antiquariat erhältlich, weil «die Betriebsamkeit der Badeverwaltung immer wieder dafür gesorgt hat, daß das kleine Büchlein des berühmten Mannes über die vortrefflichen Wirkungen der Therme zu Reklamezwecken immer neu gedruckt in Umlauf kam» (Sudhoff IX, 31). Paracelsica intra vitam waren auch die bekannten prognostischen und mantischen Schriften, die "Practica», von denen unser "Brattig» abstammt. Eine davon, die "Außlegung deß Cometen / erschienen im Hochbirg / zu mitlem Augsten / Anno 1531» ließ der Prediger Leo Jud in Zürich 1531 drucken. Sie ist am Ende unseres vierten Paracelsus-Bandes faksimiliert aus der zweiten Huser-Ausgabe in Folio bei Zetzner Straßburg 1616. Doch kehren wir zum Thema zurück, nachdem wir Hohenheim ein kurzes Weggeleite behufs zeitlicher Normierung für das zu analysierende Dokument gegeben haben.

1531/32 finden wir Paracelsus in St. Gallen, wo er sich zweifellos niedergelassen hätte, wenn ihn nicht die Reformationswirren in den Jahren der Kappelerkriege vertrieben hätten wie die Bauernunruhen aus Salzburg 1525, der Ruf nach Basel aus Straßburg 1526 und sein reformerischer Archeusgeist aus Basel 1528. Als Wanderprediger und Arzt verbannte er sich hernach in die Wüste oder in die Einöd Appenzell, wo er die Abendmahlsschriften schrieb nebst andern Theologica, die, noch unediert, in Sudhoffs Handschriftenband exzerpiert sind. Als ihn die «Hundsketten» 
und das Joch des täglichen Pfluges zu schwer drückten im Appenzellerland, schüttelte der Prophet den Staub von den Füßen, wanderte ins Rheintal hinunter gen Innsbruck, von da nach Sterzing, wo 1534 die Pest herrschte, und über den Brenner nach Meran durchs Veltlin, das damals mit Bormio, Cläven, Sondrio, Tirano bis zum Alpenwall südlich der Adda oder Etsch zu Graubünden gehörte, hernach über die Bernina nach St. Moritz, wo er den Sauerbrunnen beschrieb, die noch heute nach ihm benannte Paracelsus-Quelle Nr. II. Dann gings über das Albeli, d. h. die Albula, 1535 nach Tiefencastel und nach dem Bad Pfäfers, wohin der Gnädige Herr Abt Johann Jakob Russinger den berühmten Arzt entboten hatte zur Heilung seiner gichtischen Gebresten oder Tartara. Eigens verfaßte Hohenheim für «Euer Gnoden» ein handschriftliches Consilium. In keinem paracelsischen Schriftwerk können wir die unverfälscht schwyzerische Ursprache Hohenheims unverwässerter studieren als in diesem einzigartigen Consilium, das, in der Schweiz 1535 geschrieben, nie von einem deutschen Kopisten adaptiert oder verballhornt worden ist. Als Illustration einer solchen Adaptation, die in unseren Tagen dem verdienstvollen Paracelsisten B. Aschner 1928 passiert ist, mag folgendes gelten. In einem Consilium kopiert Huser in seinem V. Band, S. 117, die Handschrift Paracelsi richtig: "Nit Schlaftrünk tun/kein Abentürty», was der Wiener Aschner in seinem II. Band, S. 473 folgendermaßen glaubt übersetzen zu dürfen: «Ihr sollt keinen Schlaftrunk einnehmen und nicht auf Abenteuer ausgehen!» Selbstverständlich bedeutet das jetzt noch im Emmental gang und gäbe Wort, das auch von Jeremias Gotthelf oft gebraucht wird, aber im Berner Oberland unbekannt ist: Ürti gleich Zeche, Gelage mit Zechkumpanen, und hat, wie auch das Wörterbuch der Gebrüder J. und W. Grimm dartut, mit Abenteuer nicht das geringste zu tun.

Ein besonderer Glücksfall ließ dieses wertvolle Consilium immer im Abteiarchiv von Pfäfers sorgfältig behütet werden. Seit Aufhebung der Pfäferser Abtei vor gut hundert Jahren findet sich das Original-Consilium im sogenannten Pfäferser Archiv der Stiftsbibliothek St. Gallen. Diese paracelsische Handschrift in Schwyzertütsch wurde anläßlich der Paracelsusgedenkfeier 1941, veranstaltet von der «Vadiana» (Stadtbibliothek St. Gallen), den Besuchern in einem besondern Schaukasten erklärt. Die Handschrift wurde zuerst von Prof. Sudhoff 1889 in den Paracelsus-Forschungen II, 171-175, abgedruckt, besprochen und datiert, (vgl. auch dessen Buch über Paracelsus-Handschriften [II] 1899, S. 46). Der erste schweizerische Paracelsus-Biograph Prof. P. R. Netzhammer, Erzbischof, 
gest. 1945 auf der Rheininsel Werd, hat von den Blättern 401 und 402 des Tom. XXVII des sogenannten Pfäferser Archivs der Stiftsbibliothek St. Gallen ein Faksimile anfertigen lassen, das er als Probe von Hohenheims Handschrift S. 51 reproduziert. Leider wird nur der Anfang des Consiliums wiedergegeben wie auch im IX. Band Sudhoffs, wo übrigens in meiner Ausgabe die auf S. 33 versprochene «Tafel» als Probe des Anfangs der Originalaufzeichnung fehlt. Deswegen ließen wir für unsern VI. Paracelsus-Band ein neues und schärferes Faksimile herstellen als das uns vorliegende von Netzhammer. Zu Dank sind wir Prof. Sudhoff verpflichtet für seine Übertragung und Mitteilung in seinem IX. Band 663/665, die wir unter Vergleichung der nicht leichten paracelsischen Originalhandschrift hier zur Analyse benutzen. Im textkritischen Apparat bemerkt Sudhoff IX, 700 zum Consilium für den Abt Russinger: Alle diese Ergänzungen (wegen Abnützung des Papierrandes) von seiten einer Pfäferser Hand (auch im Faksimile ersichtlich) sind Zeichen der Sorgfalt, welche man auf diesen Paracelsischen Ratschlag verwendete und damit des Wertes, welchen man diesem Consilium für den Herrn Abt beimaß.

Meine kurze Analyse bezieht sich hier speziell auf das unverfälschte Schwyzertütsch, das wir nirgends so eindeutig und klar studieren können wie in diesem Consilium. Interessant ist auch zu hören, daß leichte österreichische dialektische Farbtöne einfließen. Überall aber muß man feststellen, daß das erste Vaterland Hohenheims, Helvetien, sprachlich dominant regiert, während das "zweite Vaterland», Kärnten oder Österreich (wie er es selbst benennt), nur leicht durchklingt, speziell in der Umlautung des a zu o. Beispiele: Eur Gnoden, Moß Wein, Oderlessin (Aderlassen), lossen machen, nochfolgend, geton. Dieses Consilium vermittelt uns also nicht nur die Schreib-, sondern auch die Sprechweise Paracelsi wie eine Grammophonplatte.

Typische Beispiele, daß das in der Jugend gehörte und angelernte Schwyzertütsch zeitlebens und unvergeßlich bei Paracelsus lebendig war, beweisen folgende Ausdrücke und Wendungen (Schreibweise Sudhoffs): des magens kelti(n); fluß vom ho-upt; ist wither ander arnzni nit zu gebruchen. Man beachte, daß Paracelsus selbst immer «nit» oder nüt für nicht schreibt, während schon Huser meistens nicht einsetzt. Ferner: all jor zwei mol; einmol; nüen mon; die arzny innemen, in win; doruf fasten; iij d.h. 3 stund, nicht Stunden, wie wir heute noch im Schweizer Dialekt sagen; ein erbsbrü; uf das wermist, so E. Gnoden erliden mag; wird den magen dowig, d. h. verdauend machen (däuig); o-uch für auch, spis, zit, uf 
den früling; uf dem zedeli; al nacht. Ganz charakteristisch ist auch die boumnuß wie das o-uch; den winter us; ho-uptfluß; ein gsunderer kopf; al winter gebruchen; den nechsten meien. Lossen machen klingt wieder österreichisch und wird meistens von den deutschen Kopisten mit machen lahn übertragen. Ebenso ist ostisch gefärbt: dorin, während «disse krütter» wieder ganz schweizerisch klingt, vor allem unser kabiskrut, die ru-eben. So oft «Eur gnod» in dis bad geht ist wieder gemütlich wienerisch, das im folgenden gebrotten (statt gebraten) schnitten verwandt ist mit unserem Brôte, wo wir das a auch in o mutieren, wie auch in jor statt Jahr oder mol statt mal. Nur mutiert der Österreicher konsequenter als der Schweizer. Jez uf den nechsten herbst klingt wieder ächt schwyzertütsch wie auch die folgenden Ausdrücke: wiß si; in mosts wis. Dorzu henken disse nochfolgenden stuck: verbindet beide Mundarten miteinander, während sekli, win, lebern, lib, recholterberi, negeli, muscatpluest rein schweizerisch klingen. Moß statt Maß und oderlessin für Aderlassen sind wieder ostisch gefärbt, während uf, all jor, abwexlen, gegen sumer, mon, uf der siten do die huft we dunt, nach altem bruch, der spis halben, schwini-fleisch, nit uf emol, wenig kes typisch schweizerische Ausdrucksformen sind. Beim letzten Satz ist Sudhoff ein Lesefehler unterlaufen, wenn er schreibt: «ziger schat mit» anstatt des selbstverständlichen nit, das Paracelsus immer in seiner Ursprache für nicht schreibt.

Im Latwergenrezept ist gelb gilgen wiederum schwyzerisch, während wurzen österreichisch klingt wie auch «dornoch mit $j$ moß verschumpt» wieder beide Dialekte vermengt zeigt (verschumpt $=$ verschäumt). Dorin geton klingt am Schluß wieder ostisch.

Dieses wertvolle Dokument der Stiftsbibliothek St. Gallen bietet uns also auch einen Klangfilm der paracelsischen Ursprache, der hier als solcher erstmalig kurz gewürdigt worden ist.

\section{Immatrikulations- und Testaturkunde des Tessiner Augenarztes Pietro Magistretti (1765 - I837) \\ Von Dr. med. Luigi Belloni}

Der Übergang vom 18. zum 19. Jahrhundert bedeutet einen Wendepunkt in der Geschichte der Augenheilkunde. Noch war der von Land zu Land ziehende Augenarzt, der wie ein Scharlatan auf den Märkten und öffentlichen Plätzen operierte und in dessen Händen die Augenheilkunde während der vorangegangenen Jahrhunderte fast vollständig gelegen hatte, 